\title{
THE ROLE OF COMMUNITY PARTICIPATION IN INTERMITTENT PREVENTIVE TREATMENT OF CHILDHOOD MALARIA IN SOUTH- EASTERN GHANA
}

\author{
S. K. KPORMEGBE and C. K. AHORLU \\ Noguchi Memorial Institute for Medical Research, University of Ghana, Legon, Ghana
}

DOI: http://dx.doi.org/10.4314/gmj.v48i2.1

Corresponding Author: Collins Kwaku Ahorlu

Email: cahorlu@noguchi.mimcom.org

Conflict of interest: None declare

\section{SUMMARY}

Background: Malaria remains a major cause of morbidity and mortality in Ghana. Very little attention was paid to community participation in malaria control in the past and this has affected most of the desired outcomes. The recent recognition of the importance of community participation in malaria control had informed the implementation of Intermittent Preventive Treatment of Childhood Malaria (IPTc). The intervention was implemented in the Shime sub-district of the Keta District in Ghana and this paper reports the evaluation of the community involvement aspect of the project.

Method: Semi-structured questionnaire were administered to 105 randomly selected caregivers. A focus group discussion was organized for nine Community Assistants (CAs) and 16 in-depth interviews were conducted with opinion leaders. The tools were used to investigate community attitudes towards, level of awareness and acceptance of the intervention. The benefits of the project and the preferred mode of drug delivery were also investigated.

Results: Findings indicated that, the people were aware of the project in their communities. They showed high level of participation and have accepted the intervention as their own. There was a reported reduction in hospital attendance from $52.4 \%$ to $6.7 \%$, which led to perceived savings for caregivers. The people preferred that community members deliver the drugs because they are more approachable, caring and sensitive to their health needs than the nurses.

Conclusion: The approach adopted by entrusting the intervention into the hands of the community members by allowing them to select their own CAs coupled with the observation of necessary community protocols had led to high level of participation, acceptance and successful outcome of the intervention.

Keywords: Community Participation, Intermittent Preventive Treatment of Childhood Malaria, Home Management of Malaria, Acceptability, Ghana.

\section{INTRODUCTION}

Malaria remains a major cause of morbidity and mortality in Ghana, accounting for over $40 \%$ outpatient clinic visit and about $20 \%$ death in children less than five years of age. ${ }^{1,2}$. Early recognition of suspected cases and prompt treatment is initiated at the home ${ }^{3,1}$. However, Ahorlu and colleagues observed that, the current Artemisinin Combination Therapy (ACT)for malaria treatment is not widely available for home management with the concerns that it could give rise to abuse, which may lead to the emergence of Plasmodium falciparum resistance as with chloroquine and sulphadoxine/pyrimethamine. ${ }^{1}$ Prompt and efficacious treatment and control of malaria is hardly available to people living in rural Ghana with limited access to health care services and this calls for interventions such as the Intermittent Preventive Treatment (IPT) for the vulnerable groups living in such environments.

IPT consist of administration of a treatment dose of an effective anti-malarial drug (or drug combination) at predetermined time points or intervals, to a specified at risk population, regardless of parasite burden or symptoms. ${ }^{4,5}$. The concept has recently been accepted as an important component of the malaria control strategy. ${ }^{1,6}$ Various studies indicated that IPT given with childhood vaccinations reduced incidence of first episode of malaria and also reduced severe anaemia by more than $50 \%$ during the first year of life. ${ }^{9-11}$ IPTc studies were also carried out in areas with seasonal malaria transmission and were found to be effective. ${ }^{9,12}$

A two year intermittent preventive treatment for children (IPTc) study combined with timely home management of malaria for children under five years of age also indicated that parasite prevalence reduced drastically from $25 \%$ to $3.0 \%$ at year one evaluation and further from $3 \%$ to $1 \%$ at year two. The study also showed that $13.8 \%$ of the children were febrile (axillary temperature of $\geq 37.5^{\circ} \mathrm{c}$ ) at baseline compared to $2.2 \%$ at year-one-evaluation, while $2.1 \%$ were febrile at year-two evaluation. 
The study thus concluded that, IPTc given three times a year (every four months) combined with timely home treatment of febrile malaria illness, is effective to reduce malaria parasite prevalence in children aged 6 to 60 months. ${ }^{1,6}$.

Very little IPTc studies have been evaluated from community perspectives to determine the role of community wide participation in success of IPTc implementation. The few studies on IPT and community include; Pitt et al., on Community perception of IPTc in Burkina Faso and $\mathrm{Mali}^{4}$ and Gysels et al. ${ }^{4,5}$, on Community response to intermittent preventive treatment for infants (IPTi) and expanded program of immunization (EPI) in five African settings. ${ }^{5}$ Findings of these two studies indicated that community perceptions and acceptance of IPT were widely favorable and generally accepted. Of concern to the two studies is the preference for single dose drug formulation for children under five years of age, which in their view could increase the success of IPTc interventions. ${ }^{4}$

This study investigated the role community participation play towards the success of the Shime sub-District IPTc interventions, which were published earlier., ${ }^{1,6}$ The study explored community involvement and attitude towards the project, level of awareness and acceptances as well as the perceived benefits to participating children, Parents/caregivers and the communities at large.

\section{Community participation in malaria control}

The call for Primary Health Care approached to healthcare delivery at the Alma Ata conference in 1978 brought the need for full community participation in healthcare delivery; however this was not the case in most malaria control project implementations. Though difficult to defined, Community participation is characterized by activeness, choice and the possibility of that choice being effected. ${ }^{13}$ It thus seen as "a process whereby specific group(s) living in a defined geographical area and interacting with each other, actively identify their needs and make decisions to meet them". ${ }^{13,14}$ In the context of health care delivery, health professional(s) identify a health need of a community and rally for support to address it. In the process, the community takes part in the decision-making, planning and implementation of the project. It is envisaged that when this is done, it will increase community acceptance and participation in health promotional and control activities.

\section{MATERIALS AND METHODS}

\section{The Study Site}

The Keta District is located on a narrow strip of coastal land between the Atlantic Ocean and many saltwater lagoons in southeastern part of Ghana. Out of the total surface area of about $1,086 \mathrm{~km}^{2}$, approximately $362 \mathrm{~km}^{2}$ is covered with water bodies. The district lies within Longitudes $0.30 \mathrm{E}$ and $1.05 \mathrm{E}$ and Latitudes $5.45 \mathrm{~N}$ and $6.005 \mathrm{~N}$. The district falls within the Dry Coastal Equatorial Climate with an average temperature and rainfall of about $30^{\circ} \mathrm{C}$ and $1000 \mathrm{~mm}$ respectively. The total population of the district was about 147,618 (2010 census) of which about $99 \%$ are Anlo-Ewe speakers. ${ }^{3}$

The Shime sub-District is made up of about 36 settlements of varied sizes. The people are chiefly subsistence food crop farmers, fishermen and petty traders. There are two health centers in the sub-district one of which is located in a study community (Galo-Sota). ${ }^{1}$ The study took place in four communities and these were Galo-Sota, Agbatsivi, Agortoe and Salo.

The Shime sub-District intermittent preventive treatment for children combined with timely treatment of suspected febrile malaria

For the Intermittent Preventive Treatment of Children (IPTc), $10 \mathrm{mg} / \mathrm{kg}$ body weight of Amodiaquine (AQ) and $4 \mathrm{mg} / \mathrm{kg}$ Artesunate (AS) daily (given as single dose) drug was administered to children between the ages of 6 and 60 months for three days using the directly observed therapy (DOT) principle. It is delivered every four months beginning in July 2007 (July and November 2007, March, July and November 2008 and March 2009). ${ }^{1}$ Timely home treatment was provided by Community Assistants (CAs) to participating children with febrile illness in between IPTc rounds. The treatment was based on predetermined criteria for treating suspected febrile illness as malaria and any case which did not meet these criteria was referred to the community health post for further evaluation and treatment with all expenses paid by the project.

\section{Community Entry}

The project was introduced into the sub-district through the Keta District Health Directorate. The district director of health services, who was a collaborator on the project, informed selected communities and together with community leaders planned for stakeholders' durbar in each of the four study communities. At these durbars, the project was officially introduced to the entire community members by the research team, where they took advantage to sensitize the people and asked for their support and cooperation. 
After explaining every aspect of the project, community members were given opportunity to ask questions to which appropriate answers were provided. The target population for the project was children from 6 to 60 months old.

The issue of why children alone were covered by the intervention came up for discussions and it was made known to the people that children are the most vulnerable to malaria and therefore needed protection.

\section{Community Assistants (CAs) Selection}

At the durbars, communities were asked to nominate two volunteers each to serve as project CAs who will deliver the interventions. The qualifications requested of a CA were to be able to read and write clearly, not involved in active politics and must have integrity in the eyes of the community leaders. Besides the CAs (two per community) an assemblyman (local government representative) was selected as a supervisor.

All children aged 6 to 60 months old in the chosen communities whose parents/caregivers consented participated in the project. At the beginning of the intervention, 413 children were recruited into the study and this had increased to 433 by the end of the study. No caregiver dissented participation. However, when a child attained 60 months, he/she was taken out of the study.

\section{Data collection}

This was a descriptive cross-sectional study using both qualitative and quantitative data collection techniques. Semi-structured questionnaire was administered to 105 randomly selected caregivers. Simple random sampling technique was used to select respondents from the community register compiled for the intervention and regularly updated. The four communities had a total of 351 Caregivers (Galo-Sota 114, Agbatsivi 57, Agortoe 73 and Salo 107) whose children were enrolled into the project. About $30 \%$ of Caregivers in each community were selected to participate in this study. Focus group discussion was organized with nine Community Assistants (CAs) and 16 in-depth interviews were conducted with 16 opinion leaders (four per community). FGD and in-depth interview respondents were purposively selected as key informants. All the tools were translated into the local language (Ewe) and pretested in a community with similar characteristics before the actual data collection was done.

\section{Data analysis}

Quantitative data obtained from the field were edited for accuracy, entered into the computer and cleaned for analysis. Analysis was done in EpiInfo version 3.4.1 and the result presented in simple frequency tables.
Content analysis was performed on qualitative data generated to identify representative narratives for presentation. Significant observations made on the field were also reported from field notes. The quantitative and qualitative findings were triangulated to complement each other.

\section{Ethical considerations}

Respondents were informed about the aims and objectives of the study and oral informed consent was obtained from each respondent. Also, consent was obtained from each community at a community durbar, where the leadership of the communities (both traditional and political) pledged their supports for the project. The project was reviewed by the Institutional Review Board (IRB) of the Noguchi Memorial Institute for medical Research, University of Ghana Legon.

\section{RESULTS}

\section{Demographic characteristics of respondents}

The ages of opinion leaders interviewed were between 40 and 70 years. They were parents / caregiver, grandparents and great-grandparents of children participating in the study. The caregivers were aged between 20 and 42 years with majority $(81.9 \%)$ being between 18 and 33 years. Most of them (91.4\%) were mothers of participating children. Majority $(55.5 \%)$ of the caregivers had their education up to the Junior High School level with a considerable number (41.9\%) not having formal education. About half $(48.6 \%)$ of the caregivers were artisans doing among other things weaving of rattan mats and 'crab-traps' for sale. Others $(8.6 \%)$ were engaged in harvesting of mangrove and date-palm branches for sale. Average monthly income reported by respondents was about $\mathrm{GH} \notin 100$ (about US\$69; at the rate of $\mathrm{GH} \notin 1.44$ per US\$1). Of the nine CAs only one is a female. Table 1 summarized the demographic characteristics of the respondents.

\section{Awareness and participation}

Opinion leaders interviewed reported that they were involved in mobilizing community members for the commencement of the project. For caregivers, 75 (71.4 $\%$ ) of them became aware of the project through community sensitization durbars. The rest were not in the community when the project commenced but were informed about it upon their return by either a family member or a community assistant. Over half (53.3\%) of the caregivers had only one child (Table 1). Caretakers who have more than two children in the study reported that they were caring for either their husband's children from another woman or were caring for their nieces and nephews in addition to their own children. 
About $44.8 \%$ of the children were between one and three years, $35.2 \%$ were between three and five years whiles $20.0 \%$ were between six and twelve months old.

Table 1 Socio-demographic characteristics of Respondents

\begin{tabular}{|c|c|c|}
\hline & $\begin{array}{l}\text { Frequency } \\
\mathrm{N}=105\end{array}$ & Percentage \\
\hline \multicolumn{3}{|l|}{ Age groups } \\
\hline $\begin{array}{l}18-25 \\
26-33 \\
34-41 \\
42-49\end{array}$ & $\begin{array}{l}53 \\
33 \\
17 \\
2 \\
\end{array}$ & $\begin{array}{l}50.5 \\
31.4 \\
16.2 \\
1.9 \\
\end{array}$ \\
\hline \multicolumn{3}{|c|}{ Highest level of education } \\
\hline $\begin{array}{l}\text { Junior High School } \\
\text { No Formal Education } \\
\text { Vocational }\end{array}$ & $\begin{array}{l}58 \\
44 \\
3\end{array}$ & $\begin{array}{l}55.2 \\
41.9 \\
2.9\end{array}$ \\
\hline \multicolumn{3}{|l|}{ Occupation } \\
\hline $\begin{array}{l}\text { Artisan } \\
\text { Petty Traders } \\
\text { Fishing } \\
\text { Others } \\
\end{array}$ & $\begin{array}{l}51 \\
41 \\
4 \\
9 \\
\end{array}$ & $\begin{array}{l}48.6 \\
39.0 \\
3.8 \\
8.6 \\
\end{array}$ \\
\hline \multicolumn{3}{|c|}{$\begin{array}{l}\text { No. of Children under five per respondent enrolled on } \\
\text { the project }\end{array}$} \\
\hline $\begin{array}{l}\text { Those with one child } \\
\text { Those with two children } \\
\text { Those with three or more } \\
\text { children }\end{array}$ & $\begin{array}{l}56 \\
40 \\
9\end{array}$ & $\begin{array}{l}53.3 \\
38.1 \\
8.6\end{array}$ \\
\hline
\end{tabular}

\section{Mode of delivering the interventions}

Respondents were satisfied with the mode of delivering the interventions to the children. They reported that drugs were stored in different containers, picked with spoon in most cases but in few cases by hand, after which the container is covered. CAs then dissolved the tablets in water for the children to drink. Most respondents $(98.1 \%)$ said they liked the way CAs went about the drug administration. When the question was asked "who will you like to administer the drugs to your child if the project is starting again? Almost all respondents $(97.1 \%)$ preferred drug administration by CAs to health workers or nurses for various reasons, among them were that health workers/nurses are insensitive to their health needs, arrogant and disrespectful to them. According to a thirty year old mother of two "...they (the nurses) just don't care about us..."

\section{Adverse drug reaction}

On adverse reaction to the drugs, $66.7 \%$ of the caregivers disclosed that their children had never reacted to the drugs. The rest 35 (33.3\%) said their children had long hours of sleep and dizziness, which was considered as side effects. In the words of a twenty five year old mother: “...my child tends to eat very well when given the drugs and this to me is a reaction to the drugs... but it is a good reaction as he normally eat well ...to me I wished the drug is given to them on daily basis".

In response to the question "what actions did you take to deal with your child's reactions to the drugs", 32 $(91.4 \%)$ out of the 35 caregivers, who reported adverse reaction, said they sought advice from the community assistants. The rest send their children to the clinic or gave them herbal medicine.

\section{Perceived benefits of the IPTc intervention}

Opinion leaders interviewed called for the continuity of the project in the intervention communities and in addition, extend it to nearby communities for the simple reason that it has brought them a great relief. Majority $103(98.1 \%)$ of caregivers also expressed the need for the project to continue after the initial two years. CAs reported that childhood malaria was a major problem in the communities, which was taking a lot of time and money to care for children with febrile illnesses, especially fever, before the introduction of the intervention. They maintained that the intervention has taken care of that for caretakers to a large extent. This position was captured in the words of one community assistant from Agbatsivi during an FGD, when he said:

"...the malaria situation has drastically reduced to the extent that caregivers hardly go to the clinic and hence saves time and money for other pressing needs. In this regard, I think that the project has been beneficial to both individuals and the communities..."

The perceived benefit of the project to the individual and the community was also captured in the following observations by a sixty five year old male opinion leader from Salo:

"...the project is beneficial to the children because it makes their bodies very strong to fight the asra disease (febrile malaria) and other health conditions. Now we rarely take our children to the clinic and that among many other things is the benefit of the project to us..."

A fifty four year old female opinion leader from Agbatsivi had this to say;

"...when a child falls sick, it is the parents, especially the mother that suffers. If a child is healthy and free from illness, the entire family benefits...the family then have time to work and save money for other things..."

About $97.1 \%$ of the caregivers said their children were in good health and this affords them time to attend to other needs. A twenty eight year old caregiver at GaloSota has this to say:

"... a healthy child stays-off enough to play. We then have time for daily activities. ...the health of the chil- 
dren in the community has improved because they are healthier than they were before the introduction of the project... I don't know the last time I paid hospital bills for my brother's daughter who is participating in the project".

Most caregivers expressed the view that their children no more suffer from asra (febrile malaria) and convulsions. Over half, 55 (52.4\%) of the caregivers reported at baseline that they often take their children with fever (asra) to the hospital but at the time of this data collection, only $6.7 \%$ said they had often taken their children with fever to the hospital in the past 12 months. Asked why they do not take their children/wards to the hospital often? The responses were almost unanimous that "the children don't fall sick these days because of the project".

Communities' relationship with community assistants Opinion leaders interviewed expressed the view that, there was harmonious relationship between community members and caregivers on one hand and CAs on the other hand. Also, all the caregivers interviewed reported that CAs took good care of the children and were meeting their expectations, by moving from house to house to administer the drugs. It was also reported that during IPTc rounds, CAs educate caregivers about the health of the children and how to protect them by making them sleep under treated bednets.

A twenty two year old mother of two from Salo said: "... as members of this community, any misbehavior on their (CAs) parts that affect the project negatively also affects them ...hence they (CAs) behaved well".

About $82.1 \%$ of the caregivers reported that they have very good relationship with the CAs with $14.7 \%$ of them saying it was good. The Community assistants were happy and proud to be associated with the project.

A 30 year old female CA at Agortoe said, “... we are happy to be serving because the project belongs to the community, ...our people entrusted the success or failure of it into our hands... and we don't want to disappoint them... failure may also affect us personally because some of us have our children, nieces and nephews in the study ...."

To a 35 year old male CA at Salo "...we are proud to be contributing towards the health and welfare of the children in our communities...". It came to light that CAs were very busy only during IPTc rounds, which comes off every four months and they were provided with bicycles to aid their movements in their various communities.

\section{Acceptance of the intervention}

Most of the opinion leaders interviewed maintained that, the project was well introduced into the community. To them the project team has observed the necessary community protocols and allowed the local people to fully participate in the project activities.

For a fifty-one year old female opinion leader at $\mathrm{Ag}$ ortoe:

"...unlike other projects which comes from the district without our consent, this one has involved all of us. The necessary protocols and hierarchy of power in the community were observed... as a result, we accepted the project as our own... and see to it that all children between six and sixty months old were part of the project...".

\section{DISCUSSIONS}

This study investigated the role of community participation in project acceptance and successful implementation. It also looked at the level of communities' participation in the IPTc combined with timely home treatment project implemented in the Shime subdistrict of the Keta district in Ghana. Findings indicate that almost everybody in the communities contributed towards the success of the intervention. They fully participated in mobilizing and encouraging caregivers to get their children into the study.

The clinical and parasitaemia findings from the intervention were reported else where ${ }^{1,6}$ The success of the project viewed in terms of acceptance and involvements was made possible by the commitment of the people to make it works, and community durbars held to promote active interactions among stakeholders and the communities at large. The observation of community protocols as well as insulating the project from all political influences made it acceptable to all shades of political groupings in the communities.

Several studies see Home-based management of malaria. $1,6,8,11,15,18$ as a major tool for winning the fight against malaria, especially among the vulnerable groups, but for this to be successful, it would require conscious efforts to integrate community members fully into the design, planning and implementation of this valuable interventions.

Preference of CAs to health workers to administer the IPTc may be driven by the fact that they (CAs) were nominated by communities themselves to deliver the interventions and these are people that are trusted by community members and were considered respectful. On the other hand they considered health workers as insensitive, arrogant and disrespectful. 
Although, some of these accusations of the health workers may be misplaced, it once again called into question the attitude of our health care providers, especially in rural public facilities. However, we must acknowledge that some of this providers work under very trying conditions and are often overwhelmed by their daily workloads.

The findings however contradict similar studies in Burkina Faso and Mali where some respondents expressed lack of confidence in the Community Health Workers (CHWs) on the grounds that some are illiterates and did not have experience in the equipment for weighing the children. The study indicated that, involvement of nurses was more likely to increase acceptability of the IPTi in the sense that, nurses are trusted by the people. ${ }^{4}$

This seemingly contradiction could be explained in terms of the fact that the current study permitted community members to select their own preferred persons for training as assistants while the studies in Mali and Burkina Faso made use of existing CHWs.

CAs in this study were motivated by the new social status that they enjoyed in the community as people with skills to manage malaria in children. Improvement in social status of community volunteer was also documented in a study in Thailand. ${ }^{13,16,17}$ Even though nurses did not participated in this study to allow for effective comparative analysis, Kweku and colleague however observed that both systems (community and facility base delivery) achieved more than $60 \%$ coverage for all four courses and over $80 \%$ coverage for 3 or more courses. ${ }^{18}$ Although a facility-based delivery system had a relatively high coverage $(86.6 \%)$ a substantial proportion of children would not have access if IPTc is delivered exclusively through the facility-based approach, especially those living in inaccessible and deprived areas where the risk of malaria is greatest. ${ }^{18}$

The reported side effect of drug falls within the known side effects (lethargy, drowsiness, nausea, vomiting, diarrhoea and fever) of the intervention drugs. Caregivers approach to handling these adverse effects by returning to the CAs who in turn sought advice from the community health post for cases that they could not handle follows the study protocol. Report of children eating well after taking the drug could not be considered as an adverse effect and a similar report was made in Ghana where caregivers said they like the intervention drug on the basis that it makes their children eat well. ${ }^{7}$ To the people, this was a positive sign that the drug was working well ${ }^{4}$ in line with a common understanding of the potency of traditional remedies. This perception might have also contributed to the acceptance of the intervention.

Reduction in the rate at which children aged 6-60 months are taken to the hospital was attributed to the effectiveness of the intervention. The perceived reduction in morbidity may lead to improvement in general health and well-being of the children and the communities as a whole. This also confirmed suggestions made in earlier studies ${ }^{15,19-23}$ that the best approach to managing malaria in endemic zones is to introduce an intervention at the community level, especially for vulnerable groups and hard to reach areas. ${ }^{18}$ Benefits of improved health are enormous as children had the strength to go to school, play and stay-off their caregivers. Caregivers on the other hand were relief of caring for their sick children and thereby save time and money to meet other needs. This may in the long term improve their socio-economic status of caretakers whose children participated in the study. Opinion leaders and caretakers interviewed had wished for the continuation of the intervention and had also requested that it is expanded to cover the nearby communities. This may be an acknowledgement of their satisfaction with the interventions implemented, which was expressed in perceived reduction in childhood morbidity among the target population.

\section{CONCLUSION}

The study showed that, community participation is vital for the success of community based interventions. However, to achieve this may require full engagement of community members in the process of planning and implementation from the start of the project; it may also require entrusting the success of the project into their hands, making them to claim ownership of it. It was clearly demonstrated that allowing community members to select project assistants from among themselves has an added value of improving community acceptance and participation. The observation of existing community protocols as well as respecting established hierarchy of powers within a study community cannot be overemphasized.

\section{ACKNOWLEDGMENT}

We thank the staff of Epidemiology Department of Noguchi Memorial Institute for Medical ResearchUniversity of Ghana. Our thanks also go to the respondents, chiefs and elders of the studied communities for their support as well as all the children who took part in the IPTc intervention, without them there could be no study. Financial support for this study was provided by the UNDP/World Bank/WHO Special Programme for Research and training in Tropical Diseases (TDR). 


\section{REFERENCES}

1. Ahorlu CK, Koram KA, Seakey AK and Weiss MG Effectiveness of combined intermittent preventive treatment for children and timely home treatment for malaria control. Malaria Journal 2009; 8:292. .

2. Ghana Health Service: Final draft antimalarial drug policy for Ghana, Accra. 2004.

3. Keta District Report. Keta, 2001.

4. Pitt C, Diawara H, Oue'draogo DJ, Diarra S, Kabore' H, Koue'la K, Traore A, Dicko A, Konate AT, Chandramohan D, Diallo DA, Greenwoods B and Conteh L. Intermittent preventive treatment of malaria in children: A Qualitative study of community perceptions and recommendations in Burkina Faso and Mali. PLoS ONE. 2012; 7(3): e32900.

5. Gysels M, Pell C, Mathanga DP, Adongo P, Odhiambo $\mathrm{F}$, et al. Community response to intermittent preventive treatment of malaria in infants (IPTi) delivered through the expanded programme of immunization in five African settings. Malar $\mathrm{J}$ 2009; 8:191.

6. Ahorlu CK, Kwadwo KA, Seakey-Kwawu A and Weiss MG Two-year evaluation of intermittent preventive treatment for children (IPTc) combined with timely home treatment for malaria control in Ghana. Malaria Journal 2011, 10:127.

7. Adjei GO, Darkwah AK, Goka BQ, Bart-Plange C, Alifrangis ML, Kurtzhals JAL and Rodrigues, OP. Parents' perceptions, attitudes and acceptability of treatment of childhood malaria with artemisinin combination therapies in Ghana. Ghana Medical Journal 2008; 42 (3): 99-106.

8. Agyepong IA. Malaria: ethnomedical perceptions and practice in an Adangbe farming community and implications for control. Social Science \& Medicine, 1992; 35(2),131-137.

9. Dicko A, Sagara I, Sissoko MS, Guindo O, Diallo AI, Kone M, Toure OB, Sacko M and Doumbo OK. Impact of intermittent preventive treatment with sulphadoxine-pyrimethamine targeting the transmission season on the incidence of clinical malaria in children in Mali. Malar J 2008, 7:123.

10. Schellenberg D, Menendez C, Kahigwa E, Aponte J, Vidal J, Tanner M, Mshinda H, Alonso P. Intermittent treatment for malaria and anaemia control at time of routine vaccination in Tanzania infants: a randomised, placebo-controlled, doubleblind trial. Lancet 2001, 357:1471-1477.

11. Massaga JJ, Kitua AY, Lemnge MM, Akida JA, Malle LN, Ronn AM, Theander TG and Bygbjerg IC. Effect of intermittent treatment with amodiaquine on anaemia and malaria fevers in infants in Tanzania: a randomised placebo-control trial. Lancet 2003, 361:1853-1860.
12. Cissé B, Sokhna C, Boulanger D, Milet J, Bâel H, Richardson K, Hallett R, Sutherland C, Simondon $\mathrm{K}$, Simondon F, Alexander N, Gaye O, Targett G, Lines J, Greenwood B and Trape JF. Seasonal intermittent preventive treatment with artesunate and sulfadoxinepyrimethamine for prevention of malaria in Senegalese children: a randomised, placebo-controlled, double-blind trial. Lancet 2006, 367:659-67.

13. Heggenhougen $\mathrm{KH}$, Hackethal V and Vivek,P. The Behavioural and Social aspect of malaria and its control: An Introduction and Annotated Bibliography. WHO/TDR/STR/SEB 2003; 03.1.

14. Bermejo A, Bekui A. Community participation in disease control. Social Science \& Medicine, 1993; 36, 1145-1150.

15. Ahorlu CK, Koram KA, and Weiss MG. Children, pregnant women and the culture of Malaria in Two Rural Communities of Ghana. Anthropology and Medicine, 2007; 14(2):167-181.

16. Okanurak K, Sornmani S. Community participation in a malaria control programme. In: Sornmani S, Fungladda W, eds. Social and Economic Aspects of Malaria Control. 1991; 100-114. Bangkok, Thailand, Faculty of Tropical Medicine, Mahidol University.

17. Okanurak K, Sornmani S. Community participation in the malaria control program in Thailand: a review. Southeast Journal of Tropical Medicine and Public Health, 1992; 23(Supplement 1):36-43.

18. Kweku M, Webster J, Adjuik M, Abudey S, Greenwood B and Chandramohan D. Options for the delivery of intermittent preventive treatment for malaria to children: a community randomised trial. PloS ONE 2009, 4:e7256.

19. Pagnoni N, Convelbo N, Tiendrebeogo J, Cousens $\mathrm{S}$ and Esposito F. A Community-based programme to provide prompt and adequate treatment of presumptive malaria in children. Transactions of the Royal Society of Tropical Medicine and Hygiene 1997; 91:512-517.

20. Dunyo SK, Afari EA, Koram KA, Ahorlu CK, Abubakar I and Nkrumah FK. Health centre versus home presumptive diagnosis of malaria in southern Ghana: Implications for home-based care policy. Transactions of the Royal Society of Tropical Medicine and Hygiene 2000; 94: 285-288.

21. Kidane $\mathrm{G}$ and Morrow RH. Teaching mothers to provide home treatment in Tigray, Ethiopia: A randomised trial. Lancet 2000; 356:550-555.

22. Sirima SB, Konate A, Tiono AB, Convelbo N, Cousens $\mathrm{S}$ and Pagnoni F. Early treatment of childhood fevers with pre-packaged antimalarial drugs in the home reduces severe malaria morbidity in Burkina Faso. Tropical Medicine and International Health 2003; 8: 133-139. 
23. Nsuugwa-Sabiiti J, Kallander K, Nsabagasani X, Mamusisi K, Pariyo G, Johansson A, Tomson G and Peterson S. Local fever illness classification:
Implications for home management of malaria strategies. Tropical Medicine and International Health 2004; 9 (11):1191-1199. 